\title{
Los inicios de la meteorología de Gustav Hellmann
}

\author{
Aura Lucía Prieto-Wilches, Sergio Bolaños-Cuéllar, Joaquín Pelkowski* \\ Facultad de Ciencias Humanas, Departamento de Lenguas Extranjeras y Facultad de Ciencias, \\ Departamento de Geociencias Universidad Nacional de Colombia
}

\begin{abstract}
Resumen
Son muy escasas, por no decir inexistentes, las exposiciones en castellano del desarrollo histórico de la meteorología como saber milenario acerca de los procesos que tienen lugar „en lo alto“ (ta meteora). Ni siquiera se dispone de traducciones de las mejores sinopsis que se han escrito en otros idiomas. El padre de la historiografía de la meteorología, Gustav Hellmann, entre sus varias contribuciones imperecederas a la historia de la meteorología, publicó un ensayo en inglés, y luego en alemán, de cómo evolucionó el saber meteorológico en su fase precientífica. Ofrecemos al lector hispanohablante la traducción, con algunas notas, de ese ensayo clásico sobre el desarrollo de la meteorología, desde las épocas babilónicas hasta el comienzo de la Revolución Científica, cuando se crearon los medios instrumentales para cuantificar los procesos atmosféricos. (C) 2017. Acad. Colomb. Cienc. Ex. Fis. Nat.
\end{abstract}

Palabras clave: Historia de la meteorología; Parapegmas; Astrometeorología; Proverbios meteorológicos.

\section{The dawn of meteorology}

\begin{abstract}
Very little has been written in Spanish about the evolution of meteorology as a corpus of knowledge about the processes taking place „aloft“. Not even the few good historical surveys in other languages have found translators into Spanish. The father of meteorological historiography, Gustav Hellmann, among his many lasting contributions to the history of meteorology, published, first in English, then in German, an essay about how the wisdom of meteorological ideas evolved in the prescientific phase. We offer the Spanish-speaking reader a translation, with a few notes, of that classic essay on how meteorology came to be developed, from Babilonian times to the Scientific Revolution, when instruments were devised that allowed quantification of atmospheric processes. (C) 2017. Acad. Colomb. Cienc. Ex. Fis. Nat.
\end{abstract}

Key words: History of meteorology; Parapegmata; Astrometeorology; Weather proverbs.

\section{Introducción}

La meteorología es un cuerpo de conocimientos sobre los procesos atmosféricos. Como tal, tiene una historia cuyos remotos inicios se desdibujan en la penumbra del nacimiento de las antiguas culturas del mundo. Su larga historia ha sido esbozada en algunas pocas obras, no más de media docena, sobre todo en alemán e inglés (una siendo una traducción del ruso), con sendas obras panorámicas en francés e italiano. En español no conozco ninguna, ni siquiera traducida. Claro está que las hay, aquellas obras monográficas sobre ciertos temas limitados con relación a la meteorología (por ejemplo, los graniceros mexicanos, y la meteorología chibcha). Pero en términos generales, es notable el hecho de que, comparada con otras ciencias, la meteorología no disponga de una nutrida selección de historias como es el caso en física y matemáticas, o química e hidrología.

El ensayo sobre el desarrollo de la meteorología hasta la invención de los instrumentos meteorológicos en el siglo XVII que ofrecemos al lector de habla hispana a continuación, en su primera traducción al castellano, pese a que ya tiene más de un siglo de existencia, no ha perdido su actualidad. Al contrario, Las publicaciones sobre la meteorología antigua y medieval no han hecho sino multiplicarse en las últimas décadas.

A iniciativa de Aura Lucía Prieto, en búsqueda de un tema meteorológico para una traducción como tesis de grado, propuse el ensayo de Hellmann que se ofrece a continuación, por ser de interés para una comunidad creciente de meteorólogos hispanohablantes.

Como la información sobre la vida y obra de Gustav Hellmann es escasa en cualquier idioma, no estará de más anteponer una semblanza de uno de los primeros meteorólogos de "pura cepa".

\footnotetext{
*Correspondencia:

Joaquín Pelkowski, jo-ellen.pelkowski@t-online.de

Recibido: 21 de septiembre de 2017

Aceptado: 30 de septiembre de 2017
} 
Gustav Hellmann nace en 1854, en la ciudad de Löwen, Silesia, en una provincia que en ese entonces era prusiana y hoy es una región al oeste de Polonia. Cursa sus estudios en la capital de Silesia, Breslavia, en Berlín y Gottinga. Tenía el don de las lenguas, y si no se decantó por la filología clásica, en boga en esa época y esa zona, fue gracias a unas conferencias públicas, pronunciadas regularmente en Berlín por el físico-meteorólogo Heinrich Wilhelm Dove (18031879), quien fuera elogiado a su muerte por algunos colegas y diarios como el padre de la meteorología moderna. Su "ley de la tempestad" era bien conocida por los meteorólogos decimonónicos. A diferencia de los físicos o químicos que se dedicaban a la meteorología, y en una época en que no existía la meteorología como rama independiente de las ciencias, Hellmann siempre se sintió como un meteorólogo de incontaminada vocación. Una vez graduado, emprende una gira de formación profesional en distintos países, incluyendo España. En San Petersburgo, en el renombrado observatorio geofísico central, colabora, sin remuneración, con el climatólogo y diseñador de instrumentos suizo Heinrich Wild (1833-1902), gran aficionado a la precisión de las medidas e instrumentos meteorológicos. Se convirtió en el modelo cuyo ejemplo Hellmann buscaba emular en adelante.

Entra a trabajar como asistente en el Instituto Prusiano de Meteorología en 1879. Tras la muerte del director Dove, y la muerte prematura del sucesor, Hellmann es nombrado director provisional en 1882, cargo que desempeñó con destreza hasta 1885, cuando el físico Wilhelm von Bezold (1837-1907) es nombrado oficialmente director. Von Bezold era físico, volcado a regañadientes hacia la meteorología. En su capacidad de meteorólogo funda la termodinámica atmosférica como la conocemos hoy. Sus publicaciones acusan un carácter netamente teórico. Introdujo el término de temperatura potencial, y los conceptos de humedad específica y razón de mezcla, por ejemplo, o de la luz purpúrea del crepúsculo, además de las isobrontas, o líneas de percepción simultánea del trueno en superficie. El instituto es sometido a una reorganización sustancial, quedando Hellmann a cargo del departamento de climatología y meteorología general, así como de la biblioteca. Se encarga de la red de estaciones pluviométricas en tierras prusianas, de mejorar los hábitos de observación, y de desarrollar instrucciones para los observadores. Estima de suma importancia el carácter de un observador meticuloso y concienzudo, buscando siempre el contacto con éste. En 1929 se acuña una medalla en su honor, para honrar a los observadores sobresalientes y dedicados, pero hasta su descontinuación en 1944 no fueron muchos los recipientes beneméritos. Hellmann diseña un nuevo pluviómetro, entre otros aparatos afines y autorregistradores, aún utilizado en Alemania y en otros países. En 1883 publica un valiosísimo repertorio de la meteorología alemana, un volumen que contiene las contribuciones a la meteorología en países de habla germana, muy útil para el historiador de la meteorología en general.
En el segundo Congreso Meteorológico Internacional de 1879, celebrado en Roma, Hellmann propone a los representantes de varias naciones la elaboración de una bibliografía, por países, con el objetivo de componer una bibliografía universal de la meteorología. Es aprobada la propuesta, pero el plan fracasa, debido a la falta de presupuestos para ello. Sin embargo, Hellmann no se descorazona y decide proceder con la publicación de la ya mencionada bibliografía. En España e Italia también se procede a la creación de bibliografías nacionales, pero solamente en Estados Unidos se publican, con mínimos recursos, cuatro tomos deleznables que han servido de base a una bibliografía reciente, editada en inglés.

En 1883 Hellmann hace parte del comité fundador de la sociedad alemana de meteorología, de la cual fue presidente entre 1907 y 1923. Su charla de inauguración en la presidencia, sobre los inicios de la meteorología -la que ofrecemos a continuación- se publica el año de 1908, en alemán e inglés.

La revista de la sociedad meteorológica alemana, que arranca en 1884, fusiona a los dos años de su existencia con la de la sociedad austriaca de meteorología, que había sido creada en 1866. Hasta el fin de la segunda guerra mundial, la Meteorologische Zeitschrift fue una de las más prestigiosas revistas de meteorología. Hellmann fue coeditor, junto con Julius von Hann (1839-1921), el más ilustre meteorólogo de Austria en su época, desde 1892 hasta 1907, cuando sucede a von Bezold como director del instituto real prusiano de meteorología, siendo nombrado al mismo tiempo profesor titular de meteorología en la universidad de Berlín. Hellmann reorienta el instituto para servir a los sectores sociales con intereses prácticos. A diferencia de otros servicios meteorológicos, y en concordancia con la política de v. Bezold, no fomentó el servicio de pronosticar el tiempo, pese a la creciente demanda del sector público. Es anecdótico apuntar que compartía la ética del canciller Otto von Bismarck (1815-1898), unificador de Alemania, quien opinaba que una institución estatal no podía quedar en ridículo. En Inglaterra, por ejemplo, FitzRoy (18051865), el capitán que timoneó a Charles Darwin en sus periplos científicos se arrojó a realizar pronósticos y alertas de temporales en los años 1859-1865, convencido de la utilidad de tales presagios, actividad que se descontinuó temporalmente tras su suicidio.

Hellmann comienza su larga lista de publicaciones poco antes de 1875. Hasta su jubilación (forzada) casi no hay fascículo de la Meteorologische Zeitschrift sin una contribución suya. A partir de su nombramiento en 1912 como miembro de la Academia Prusiana de Ciencias, también publica regularmente en las actas de ésta.

Como climatólogo-geógrafo que era, preparó con sus colaboradores del instituto, extensas obras de climatología, entre las que cabe citar la distribución de las precipitaciones por provincias alemanas, que culmina, en 1906, con tres enjundiosos tomos sobre la lluvia en las llanuras de la 
Alemania septentrional. Una extraordinaria creciente del Óder, importante río en Europa Central, en 1888 y 1903, con precipitaciones cataclísmicas, lanza una investigación dirigida por Hellmann sobre la situación meteorológica que acompañó a la segunda, y en 1911 publica con Elsner una magnífica obra detallada sobre la cuestión, con un atlas en folio doble marquilla de mapas sinópticos, y un tomo de texto. Publica memorias sobre varios meteoros, sus distribuciones y explicaciones, así como una clasificación de hidrometeoros, el Nuevo sistema de hidrometeoros, en 1915, en gran pate adoptado por la comunidad meteorológica. Ya en 1893 Hellmann había publicado una joya bibliófila, acerca de los „cristales de hielo“, con microfotografías tomadas por Neuhaus, que revelan por primera vez en público la belleza y los detalles de los cristales de hielo, así como el hecho de que ostentan una estructura interna. Es el primer libro sobre el tema, aunque ya había aficionados dedicados a las microfotografías de cristales níveos. Es también muy valiosa por su revisión histórica de aquellos que anteriormente habían observado y dibujado cristales o copos de nieve, que va desde el siglo XV hasta finales del XIX.

Para dar una idea del carácter de los trabajos de Hellmann, nada mejor que darle la palabra a Max Planck (1858-1947), premio Nobel de física por haber establecido la ley de radiación que lleva su nombre, y de paso haber creado la física cuántica. En su función de secretario de la clase fisicomatemática de la Academia Prusiana de Ciencias, comenta en su réplica al discurso de posesión de Hellmann, que las investigaciones de éste "no son realizadas desde un punto de vista dinámico, sino estadístico", añadiendo sagazmente que "sería equivocado pensar que el método estadístico pueda llevarse a un enfrentamiento con el método físico. Más aún, si los indicios no nos engañan, el desarrollo de la rama más reciente de la física - física molecular y atómica — está pujando hacia una concepción estadística, que aspira a entender el nexo causal de los procesos elementales en virtud de la acumulación de fluctuaciones irregulares de sendos eventos". Tras señalar que no había diferencias esenciales de método debidas a las distintas escalas, hace la concesión de que el meteorólogo se encuentra en desventaja con respecto al físico, ya que aquél no puede controlar las condiciones del fenómeno bajo estudio, recalcando que, por otro lado, "el meteorólogo se encontraba en la posición venturosa de conocer con todo el detalle posible las leyes elementales que subyacen a los procesos atmosféricos: movimientos del aire, cambios de presión y de temperatura, formación de la precipitación.” Sin duda Planck compartía el entusiasmo y optimismo de Vilhelm Bjerknes (1862-1951), padre de la predicción fisicomatemática del tiempo, cuando añadía: "Sin duda es prematuro esperar que algún día se logre, gracias a una combinación de los métodos estadístico y dinámico, en el sentido de los esfuerzos de Vilhelm Bjerknes, el acercamiento al objetivo final de toda investigación meteorológica: el pronóstico del tiempo. Mientras tanto, y durante mucho tiempo más, la meteorología práctica ha de recabar y comparar datos; en este sentido ha sido usted más que nadie, quien ha sentado las bases, mediante su extenso trabajo sobre las precipitaciones en provincias prusianas, de una climatología ejemplar, incluso para otros países." En cuanto al pronóstico del tiempo, ya hicimos notar que Hellmann lo rechazaba por 'inexacto' y por ser susceptible de seria atención solamente por caracteres "románticos" como Bjerknes.

Planck asimismo hace hincapié en sus habilidades como diseñador o perfeccionador de aparatos autorregistradores, exaltando el incansable y versátil organizador de todo trabajo científico, quien domina con mano firme la maquinaria de su instituto, amén de fijarse en las actividades de otras instituciones extranjeras, que conoció en sus numerosos viajes.

Finalmente pasa a mencionar un aspecto distinto de la labor de Hellmann, que ya de por sí abarcaba tanto, llamando la atención a los trabajos de éste como historiador de la meteorología y del geomagnetismo: "La Academia no solo lo conoce como un investigador circunspecto, y como alguien que elabora el material disponible con imaginación y agudeza, sino que valora altamente el consumado experto bibliográfico, quien, en medio de sus absorbedoras tareas profesionales, aún encuentra tiempo de ocio para sumirse en los registros de épocas poco familiares, e incluso para hacerlos asequibles a través de reproducciones facsimilares."

De sus aportaciones a la historia de la meteorología, publica un último tomo en 1922. Jubilado en 1923, sigue publicando artículos sobre climatología e historia de la meteorología. Pero no realiza su sueño de juventud, cual era redactar una historia de la meteorología, pese a las reiteradas súplicas de muchos colegas que conocían sus numerosos ensayos sobre temas históricos.

No es este el lugar para evocar con justicia al gran historiador de la meteorología. Queda aún por demostrar que Hellmann merece el epíteto de padre de la historiografía meteorológica. Mi impresión es que hoy en día tanto historiadores como filólogos citan al historiógrafo, mientras que los numerosos trabajos del meteorólogo y climatólogo, si no están completamente relegados al olvido, han sido asimilados por fríos y secos registros de un servicio meteorológico. Su labor como procurador industrioso de libros le valió convertirse en epónimo sonoro de la biblioteca del Servicio de Meteorología Alemán, una de las más grandes del mundo en materia de meteorología.

Octogenario, su salud se deteriora, y Hellmann fallece a principios de 1939, antes del gran cataclismo que acabaría con la clásica erudición prusiana.

\section{Los inicios de la meteorología, por Gustav Hellmann ${ }^{1}$}

La meteorología como ciencia es joven, pero como rama del saber es muy antigua, quizá tan antigua como la humanidad.

\footnotetext{
${ }^{1}$ Conferencia dictada por Gustav Hellmann como recién elegido presidente de la Deutsche Meteorologische Gesellschaft (sociedad alemana der meteorología) en 1907, y publicada un año después en la entonces prestigiosa revista de dicha sociedad. Hellmann fue invitado por la Royal Meteorological Society a pronunciar la misma conferencia, lo cual hizo en un impecable inglés. La revista de esta sociedad publicó la versión, ligeramente modificada, también en 1908, bajo el título The dawn of meteorology.
} 
De hecho, los inicios de la meteorología deben buscarse desde el momento en que la cultura humana empezó a existir. En aquellos tiempos lejanos, el hombre, cuya ocupación era la agricultura y la caza, dependía más del clima que nosotros en la actualidad y esto hizo que con frecuencia prestara atención a los fenómenos atmosféricos. Desde luego no lo hizo porque sí, ni mucho menos para indagar las leyes que rigen los fenómenos atmosféricos, sino para sacar ventaja de ellos. Quería aprender a protegerse de las inclemencias del clima, a detectar el tiempo más favorable para realizar sus actividades y a encontrar las condiciones atmosféricas más adecuadas para el éxito de su cosecha.

Las experiencias vividas al respecto se heredaron y se multiplicaron de generación en generación, conformando desde el principio una base sólida del saber popular. Se trata de la sabiduría tradicional acerca del clima, la cual pervive hoy en día y perdurará para siempre. Esta se ha expresado y todavía se expresa en forma de proverbios y reglas absolutas, pues así se graban mejor en la memoria. Una gran parte de estas sentencias sobre el tiempo se basaba, en realidad, en la observación de los fenómenos climáticos naturales, tales como los vientos, las nubes y fenómenos luminosos en el cielo. Sin embargo, estas aseveraciones provenían a menudo de conclusiones y asociaciones de ideas erróneas y correspondían al bajo nivel intelectual de la formación escolar, al que se le sumaban también ideas supersticiosas.

Por lo tanto, no debemos creer que la rica variedad de proverbios o refranes respecto del tiempo que se encuentran, por ejemplo, en la Biblia, principalmente en el libro de Job, en Homero y en Hesíodo, escritores del siglo VIII a.C. se hubieran originado en esa época en Palestina o en Grecia. La familiaridad del pueblo con estas sentencias y reglas del tiempo, y el uso informal de ellas por parte de los escritores lo que nos muestra, más bien, es que ya debían ser vistas como un acervo ancestral de la cultura de entonces. Ciertamente tenemos una buena razón para creer que parte de la sabiduría tradicional y de las supersticiones acerca del tiempo provienen incluso de la cuna del indoeuropeo.

En dos casos he podido probar claramente que las creencias modernas populares acerca del tiempo son muy antiguas. Se trata precisamente de supersticiones sobre el tiempo en ambos casos, puesto que nada se arraiga tan firmemente en la imaginación popular como lo hace la superstición misma.

En toda Europa, como es sabido, se atribuye especial significado de pronóstico a las doce noches, o los doce días de comienzo de año, conocidas como cabañuelas, que después, bajo la influencia de la iglesia cristiana, se contaron a partir de Navidad. La temperie en estas noches o días había de coincidir con las de los doce meses del año que iniciaba. Además de la tradición que aún hoy en día se conoce popularmente, esta creencia se puede rastrear en la literatura hasta el siglo XV; incluso antes es posible encontrarla en numerosos manuscritos del siglo IX. Además, no era desconocida al padre de la iglesia escocés, Beda el
Venerable ${ }^{2}$, en el siglo VIII. Asimismo, la recopilación greco-bizantina sobre la agricultura, La Geopónica ${ }^{3}$, escrita en el siglo VI, nos enseña que Demócrito en el siglo V a. C., ya la conocía de una forma un tanto diferente. Si además nos enteramos, a través de los sanscritistas, que los textos védicos también conocen "las doce noches", entendiéndolas como una alegoría del año venidero, parece muy probable que esta superstición relativa al tiempo atmosférico tenga un origen indoeuropeo antiguo. Los pueblos europeos trajeron esta superstición desde el lugar de origen que compartían. No solo se extendió esta creencia desde la India hacia el occidente, donde fue difundida y conservada por los indoeuropeos, sino que también se desplazó hacia el oriente. Así, aún hoy en día se celebra en China una tradición en la noche de año nuevo que corresponde del todo con la concepción indoeuropea del significado de pronóstico que tiene el comienzo de año para el tiempo del año que inicia.

Otra superstición respecto del tiempo nos lleva a la Antigua Babilonia como lugar de origen. En muchas obras antiguas de la literatura europea popular y en el libro de feria Sibyllae Prophetia, que aún se imprime en Suecia, se encuentran pronósticos de la temperie y la fertilidad para todo el año, a partir de los truenos que se oían en cada mes. Estos signos del trueno ("signa tonitrui”) se pueden rastrear hasta muy entrada la Edad Media en manuscritos en todos los idiomas de Europa occidental, y sin duda se remontan ante todo a la rica literatura sobre los "almanaques ceráunicos" o "brontologías”, en cuya redacción participaron los propios

\footnotetext{
${ }^{2}$ Beda (673-735), apodado «el Venerable» por los medievales, es la figura más destacada del monaquismo anglosajón. Monje en el monasterio de San Pablo en Jarrow, Inglaterra, historiador consagrado al estudio de la Biblia, escribió la famosa Historia eclesiástica de la nación inglesa y una obra muy leída en el medioevo, su De rerum natura, basada en el modelo de la enciclopedia de Isidoro de Sevilla (véase la nota 20). Borges, en su deleitable obra sobre la literatura germánica, juzga que las obras de Beda son "estudiosos epítomes, de gran erudición, de escasa originalidad, pero saturados de claridad y mansedumbre”. Cf. Borges, Jorge Luis, 1978: Literaturas germánicas medievales. Con la colaboración de María Esther Vázquez. Emecé editores, Buenos Aires.

${ }^{3}$ La Geopónica es el nombre de una colección de veinte libros sobre agronomía y agricultura escrita en griego y compilada durante el siglo $\mathrm{X}$ en Constantinopla por el emperador bizantino Constantino VII. La palabra griega geoponica ( $\gamma \varepsilon \omega \pi$ ovıкó) significa "empresas agrícolas" en su sentido más amplio. La obra abarca todo tipo de información agraria, incluida la meteorología celeste y terrestre. Se ha atribuido erróneamente al autor del siglo VII Casiano Baso. Hellmann probablemente consultó a Henricus Beckh, quien edita en 1895 el texto de Geoponica sive Cassiani Bassi scholastici de re rustica eclogae, lo que explicaría la fecha errada que indica. (Tomado de Wikipedia.)

${ }^{4}$ Un ejemplo de brontología se encuentra en el libro reciente The Religion of the Etruscans, editado por Nancy Thomson de Grummond y Erika Simon, por la University of Texas Press, 2006. Algunas apódisis son condiciones de tiempo de toda clase (v.g., gran calor, nubosidad, lluvia), pero la mayoría son consecuencias no en sí meteorológicas sino dependientes del tiempo (enfermedades, buenas cosechas, pesca maluca; ejemplo: el 12 de noviembre, si truena, muchos hombres padecerán insomnio) y algunas no tienen conexión con el tiempo (discordia civil, por ejemplo). En otra parte nos dice que generalmente soñar con un relámpago es una señal desfavorable, para algunos es buena suerte.
} 
emperadores bizantinos de los siglos IV y $\mathrm{V}$ y que debieron haber tenido una extraordinaria difusión. En un capítulo similar de la ya mencionada Geopónica se le atribuye este saber a Zoroastro. Si bien es posible que no sea el autor de esta doctrina, su nombre señala aproximadamente a la esfera espiritual de la cual procede, concretamente, del antiguo Oriente. Y, de hecho, encontré en los escritos de dos asiriólogos, Sayce en Oxford y Lénormant en París, la prueba de que esta superstición relacionada con tormentas eléctricas es de origen caldeo.

Con esto llegamos a un periodo históricamente mejor avalado, a la época de la cultura sumerio-babilónica. El estado de la meteorología como una rama del saber tuvo en ella, es decir, aproximadamente de 2 a 3 milenios a. C., un carácter fundamentalmente diferente al que tenía en los primitivos tiempos antes aludidos, en los que se originaron los primeros proverbios del tiempo.

Después de haberse dado una incipiente erudición en las castas sacerdotales, y que la observación de la bóveda celeste permitió constatar ciertas regularidades, se conectaron causalmente los fenómenos atmosféricos y las constelaciones de los astros, creándose así un sistema de consecuencias y combinaciones bastante complicado, que significó el origen de la astrometeorología. Esta constituyó verdaderamente una parte integrante de la religión asiriobabilónica y más tarde desempeñó un papel decisivo en todo el campo cultural europeo-asiático hasta el siglo XVII, e incluso hoy en día no ha desaparecido del todo5.

Gracias a los memorables descubrimientos de la Biblioteca Astrológica de escritos cuneiformes de Asurbanipal por parte de Sir Henry Rawlinson, escritos que se conservan en estos momentos en el Museo Británico y que habían sido en gran parte descifrados recientemente por R. Campbell Thompson y Franz K. Kugler, hemos podido conocer por primera vez más ampliamente el sistema astrometeorológico de los habitantes de Mesopotamia, cuya existencia fue aludida muchas veces por los escritores de la Antigüedad en Grecia y Roma, pero que nunca lograron explicar con precisión.

Los registros meteorológicos de los caldeos eran completamente de naturaleza selectiva y se relacionaban, en primer lugar, con manifestaciones luminosas, en especial con los halos, de los cuales sabían distinguir el pequeño de $22^{\circ}$ ("tarbasu”) del grande de $45^{\circ}$ ("supuru”). A continuación, se prestó mucha atención a las nubes, a los vientos, a las tempestades y a las tormentas. Al parecer, estas observaciones servían poco para realizar propiamente pronósticos meteorológicos en el estable clima de Babilonia, donde no hacían mucha falta; servían más bien para profetizar

\footnotetext{
${ }^{5} \mathrm{La}$ astrometeorología tiene una larga historia, que aún continúa, con asociaciones institucionales fundadas con cierta persistencia. Véase Hellmann, Die Wettervorhersage im ausgehenden Mittelalter (La predicción del tiempo en el Medioevo tardío, del siglo XI al XV), Beiträge zur Geschichte der Meteorologie, Nr. 8, 1917; Stuart Jenks, 1983, Astrometeorology in the Middle Ages, Isis, Vol. 74, No. 2, pp. 185-210; Julio Samsó, Astrometeorología y Astrología Medievales. Publicacions i Edicions de la Universitat de Barcelona. Barcelona, 2008.
}

todo tipo de cosas buenas y malas. Los pronósticos y las profecías estaban dirigidos siempre al soberano de la nación y registraban al final, por lo general, a quien pronosticaba, es decir, la fuente en la que se apoyaba la previsión. He aquí algunos ejemplos meteorológicos:

(Thompson Nr. 137) Si un halo rodea el sol, caerá lluvia. Según Irassi.ilu.

(Thompson Nr. 248) Si una nube en el cielo se vuelve negra, soplará el viento.

Según Nabi-ahi-iriba.

(Thompson Nr.261) Si truena en Sebat, habrá una plaga de langostas....etc.

Estos son, en parte, pronósticos en el sentido de los “omina” y "portenta” romanos tardíos, que, a través de acciones sacras de los pontifices, debían ser expiados. No debo dejar de resaltar que en los antiguos textos védicos se encuentran interpretaciones de procesos meteorológicos muy parecidos, para los cuales también se precisaban los sacrificios exigidos.

Así, por ejemplo, en un comentario (Adbhutabrahmana) de Samaveda, según la traducción de A. Weber, se lee: "Después de que el aire regresa, cuando soplan los vientos en exceso, cuando se forman en las nubes rostros deformes..., cuando desde arriba se vierte lluvia de polvo, de carne, de huesos, de sangre..., cuando de noche el hombre ve un arcoiris..., los árboles transpiran sangre, en el aire aparece el palacio de un rey (probablemente, una fata morgana) y similares. Todas estas señales están consagradas a Vayu. Con la frase 'el viento trae la cura hacia aquí' que presente una ofrenda cocinada en una olla..." etc.

En las inscripciones cuneiformes, de las cuales solo se ha recuperado una parte relativamente pequeña y de las cuales muy pocas se han podido descifrar, no se ha podido encontrar hasta ahora alusión alguna a la existencia de teorías meteorológicas. Sin embargo, me parece un hecho destacable que los babilonios efectivamente utilizaran la rosa de los vientos octopartita, creada de la manera más simple a partir de la combinación de cuatro direcciones principales, en tanto que generalmente se suponía hasta ahora que solo en el círculo de Carlomagno, tal vez por iniciativa del monje Alcuino ${ }^{6}$, proveniente de York en Inglaterra, se logró el progreso en cuanto a que se usaron los nombres de las cuatro direcciones principales: norte, sur, oriente y occidente, combinadas de modo que se pudieran designar todas las direcciones del viento. En cambio, los griegos y los romanos tenían un nombre especial para cada viento, como sigue siendo la costumbre de los marineros italianos del Mediterráneo que conservan hasta hoy en día estas designaciones. Las direcciones principales babilónicas se presentan en el siguiente orden: sur, norte, oriente, occidente y se llaman šutu, iltanŭ, šadu, amurra. Al unir estos nombres

\footnotetext{
${ }^{6}$ Alcuino de York (730-804) se formó en la escuela episcopal de Jarrow, que había sido fundada por Beda el Venerable. Fue director de la escuela palatina de Carlomagno y consejero del rey en cuestiones relacionadas con la docencia, instrucción y el culto.
} 
con la conjunción copulativa “y” (en babilonio $u$ ) aparecerán las cuatro direcciones secundarias, por ejemplo: šutu u šadu = sudeste, iltanu и amarru = noroeste, etc.

Los escritos de la Antigüedad hablaban de la influencia que había tenido la astronomía babilónica sobre la griega. Sin embargo, en las concepciones meteorológicas que hallamos en la literatura griega no se hace evidente dicha influencia. Si bien es cierto que en Hesíodo se presentan diversas reglas astrometeorológicas que en parte pueden provenir del Oriente, ya desde el siglo VI a.C. los griegos parecen haber realizado observaciones meteorológicas con regularidad, y casi un siglo antes hallamos los inicios de una teoría meteorológica, resultado, ante todo, del elevado espíritu científico de los griegos.

Además de que Teofrasto (372-287 a.C.) ${ }^{7}$ hace referencia explícita a varios hombres que hicieron observaciones astronómicas y meteorológicas en diferentes ciudades de Asia Menor y Grecia, debemos deducir de la existencia de tales registros el establecimiento de aquellos parapegmata o calendarios "móviles" (de "clavija”) de naturaleza peculiar, que desde los tiempos de Metón se instalaban en columnas en lugares públicos para la instrucción general del pueblo. ${ }^{8}$ Estos, al igual que los calendarios del tiempo modernos, contenían datos meteorológicos promedio para días concretos o periodos de tiempo cuya fijación temporal se obtenía a través de la salida y puesta de las estrellas. En vista de que estos parapegmata eran diferentes en cada lugar, se deduce claramente que se basaban en observaciones particulares.

En la Figura 1 se muestra la imagen de fragmentos de dos parapegmata encontrados en Mileto, que se conservan hoy en día en el Museo de Berlín.

A continuación, se presentan algunos ejemplos de un parapegma que se atribuye al famoso astrónomo Gémino9:

31 de agosto. Para Eudoxo, sopla un gran viento y truena. Para Calipo, realizan su orto los hombros de la Virgen; cesan los etesios.

5 de septiembre. Para Euctemón, es visible el Heraldo de la Vendimia; sale la estrella Arturo y se pone la Flecha al amanecer; tempestad en el mar; vientos del sur: Para Eudoxo, lluvia y truenos.

\footnotetext{
${ }^{7}$ Teofrasto, discípulo de Aristóteles y sucesor de éste en el Liceo. Una edición y traducción reciente de la obra que Hellmann menciona es: Theophrastus of Eresos, 2007: On Weather Signs. Edited by David Sider \& Carl Wolfram Brunschön, Brill, Leiden.

${ }^{8}$ Véase Lehoux, Daryn, 2007: Astronomy, Weather, and Calendars in the Ancient World. Parapegmata and Related Texts in Classical and NearEastern Societies. Cambridge University Press, New York. Igualmente se puede consultar sobre los parapegmas la obra de Taub, Liba, 2003: Ancient Meteorology. Routledge, London.

${ }^{9}$ Para una versión castellana, véase la edición conjunta de dos escritos: Arato, 1993, Fenómenos. Géminos: Introducción a los fenómenos. Introducciones, traducciones y notas de Esteban Calderón Dorda. Biblioteca Clásica Gredos, 178. Editorial Gredos, Madrid.
}

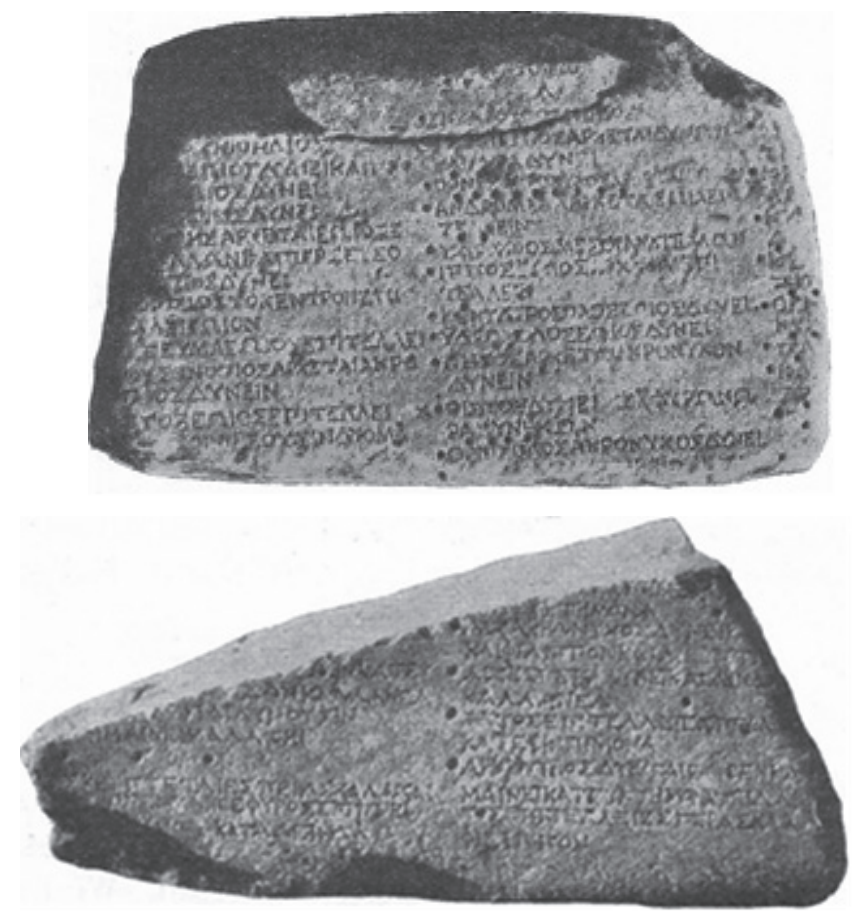

Figura 1. Fragmentos de dos parapegmata. En estas piedras de mármol se ven agujeros destinados a sostener clavijas, para fijar el principio del año y los días, que es lo que les dio su nombre, derivado del verbo griego $\pi \alpha \rho \alpha \pi \eta \gamma v v ́ v \alpha \iota=$ enclavijar, fijar dentro.

12 de septiembre. Para Calipo, pronostica cuando la Virgen está a medio salir; Arturo realiza su orto aparente. El tiempo tiende a cambiar.

14 de septiembre. Para Eudoxo, Arturo realiza su orto matutino; soplan vientos durante los 7 días siguientes; buen tiempo en general; al finalizar este período hay viento del este.

En estos parapegmas preponderan las observaciones de los vientos, lo cual no nos debe sorprender, pues los requerimientos prácticos de la navegación las exigían, en tanto que el sentido especulativo de los griegos se ha ocupado desde tiempos inmemoriales del origen de los vientos. Si bien en tiempos de Homero se entendían los vientos como seres independientes, como deidades, en el siglo $\mathrm{V}$, el filósofo natural jónico Anaximandro ${ }^{10}$ presentó por primera vez una definición científica del viento, que todavía tiene validez hoy en día: "el viento es un flujo de aire".

Así se explica el hecho de que los griegos hayan tenido veletas desde una época temprana, que representan el instrumento meteorológico más antiguo y de las que

\footnotetext{
${ }^{10}$ Anaximandro de Mileto, fl. 580 a. C., discípulo de Tales. Cabe citar uno o dos fragmentos, uno de Aecio:» Anaximandro dice que el viento es una corriente de aire en la cual las partículas más livianas y húmedas son movidas o consumidas por el sol «, y el otro de Hipólito: «Los vientos se generan al separarse del aire los vapores más livianos y cuando, al moverse, se concentran.» Véase Los filósofos presocráticos, 1978, Tomo I. Introducción general por Conrado Eggers Lan. Introducciones, traducciones y notas por Conrado Eggers Lan y Victoria E. Juliá. Biblioteca Clásica Gredos, 12. Editorial Gredos, Madrid.
} 
nos quedó, en parte, un ejemplo arquitectónico interesante en la llamada "Torre del Viento" en Atenas. Sin embargo, no quisiera creer que el fin último de esta construcción de Andrónico de Cirrestes era determinar la dirección del viento. Esta torre se encuentra en la plaza de mercado ubicada en lo hondo (agorá), directamente por debajo de la elevada Acrópolis, que por eso mismo es poco adecuada para la observación de la verdadera dirección de los vientos. Más bien parece ser que el reloj de agua o clepsidra, emplazado en el edificio, alimentado por una fuente cercana del mismo nombre, estaba destinado ante todo a indicar el tiempo (cronológico) en la muy frecuentada plaza de mercado, pero el constructor, por razones arquitectónicas y estéticas, ideó una torre de viento octogonal. Mi opinión está respaldada por el hecho de que, de acuerdo con el testimonio de Teofrasto, los astrónomos y meteorólogos de Atenas preferían realizar las observaciones no en la poco elevada ciudad, sino más bien desde la despejada colina Himeto, fuera de ella.

En aquella época, es decir, aproximadamente en el primer siglo a.C., el uso de las veletas debió haberse expandido y perfeccionado ampliamente, pues el escritor romano Terencio Varrón ${ }^{11}$, escribiendo sobre la agricultura, cuenta que ya había las llamadas "veletas continuas" en casas de campo romanas, de modo que la dirección del viento se podía observar desde las habitaciones. Siendo esto así, siempre me ha parecido muy curioso que, a pesar de ello, no conozcamos ninguna palabra griega o latina para veleta.

Las observaciones meteorológicas cuantitativas más antiguas, al parecer se realizaron en el primer siglo de la cronología cristiana en Palestina precisamente durante el periodo de lluvias, cuya medición temprana en pleno clima mediterráneo tenía importancia crucial para el éxito de la cosecha.

Al comparar en la Mishná, una recopilación de escritos judíos de aquella época, las magnitudes normales de pluviosidad para una buena cosecha con las medidas modernas en Jerusalén, se constata que concuerdan en gran medida, por lo cual se puede concluir que, desde entonces el clima de Palestina no pudo haber sufrido ningún cambio considerable en este respecto. Es bien sabido que hace aproximadamente 70 años Arago ${ }^{12}$, aduciendo razones fenológicas o fitogeográficas, llegó a la misma conclusión.

Pero también le debemos a la Antigüedad clásica los fundamentos de la invención de uno de los más importantes instrumentos meteorológicos: el termómetro. Algunos lectores pueden sorprenderse, pues incluso en la actualidad en los círculos de especialistas en ciencias naturales, todavía

\footnotetext{
${ }^{11}$ Varrón, siglo 1 a.C., autor de una obra sobre los vientos. Junto con ésta aparece el «calendario rústico» descrito por en el De re rustica, según el cual los “días” 23 de Acuario, Tauro, Leo y Scorpio marcan respectivamente los límites de la primavera, verano, otoño e invierno.

${ }^{12}$ François Arago (1786-1853), físico, astrónomo y meteorólogo francés. No apoyaba la predicción del tiempo rutinaria, por considerarla inmadura en su época.
}

persiste la idea de que en la Antigüedad no se produjeron instrumentos ni se realizaron experimentos. Cuanto más conozcamos la literatura científica y técnica de los griegos y romanos, gracias a la investigación filológica moderna, que se dedica al estudio de las prácticas y no solo a la bella literatura, tanto más debemos convencernos de que la Antigüedad en este respecto ofrece diversos y muy respetables resultados.

Entre las obras físico-técnicas de Filón de Bizancio, quien probablemente vivió en el siglo III a.C. y de Herón de Alejandría en el siglo I d.C. encontramos dos aparatos a los que debemos referirnos como prototipos del termoscopio. La descripción de Filón en su obra "De ingeniis spiritualibus" (De las máquinas neumáticas, o a presión de aire), cuya copia original en griego se perdió y de la que solo se tienen traducciones en árabe y latín, dice así en (en nuestra traducción al castellano de una traducción al alemán): «Se fabrica una esfera de plomo (eolipila) de tamaño moderado, vacía y espaciosa por dentro. No deberá ser muy delgada para que no estalle inmediatamente; ni tampoco muy pesada, pero debe estar totalmente seca para que mejor se logre su propósito. Después perfórese en la parte superior, y colóquesele un sifón que llegue casi hasta el fondo. Del mismo modo se coloca el otro extremo encorvado en otro recipiente, lleno de agua, el cual, al igual que en el primer recipiente, llegue casi hasta el fondo, para así facilitar el desagüe del agua. Afirmo que cuando se pone la esfera al sol, gracias al calentamiento parte del aire que estaba en el tubo saldrá. Esto se podrá observar porque el aire del tubo fluye hacia el agua, la pone en movimiento y provoca burbujas, una tras otra. Por el contrario, si la esfera se ubica bajo la sombra o en algún otro lugar en el cual no penetren los rayos del sol, el agua subirá a través del tubo hasta alcanzar el fondo de la esfera de plomo. Si se vuelve a poner al sol, el agua fluirá de nuevo en aquel recipiente... Y todas las veces que se repita el proceso se podrá observar este fenómeno. Incluso si se calienta la esfera con fuego o si se riega con agua caliente, se obtendrá el mismo resultado...» (Cf. Fig. 2.)

El aparato análogo de Herón, al que nombra $\lambda \imath \beta \dot{\alpha} \varsigma$ (gotera) es un poco más complicado. Gotea al estar expuesto al sol.

El tratado de Herón de las fuerzas de presión (neumática), que parece haber tenido una gran difusión manuscrita en la Antigüedad y a comienzos de la Edad Media, apareció no menos de dos veces traducido al latín y no menos de tres veces traducido al italiano, en el lapso de 18 años entre 1575 hasta $1592^{13}$, y probablemente inspiró, casi simultáneamente, la construcción del termoscopio por tres eruditos de aquel entonces, concretamente, Galileo, Porta y Drebbel, quienes alrededor de 1600 se esforzaron por construir dicho

\footnotetext{
${ }^{13}$ Hoy disponemos de traducciones a lenguas modernas. Ignoro si existe una versión en castellano, pero conozco una francesa y otra en inglés. Ésta contiene dibujos de los aparatos y de la gotera que Hellmann menciona. Se trata de The Pneumatics of Hero of Alexandria. Introduced by Marie Boas Hall, Macdonald, London \& American Elsevier, New York, 1971.
} 


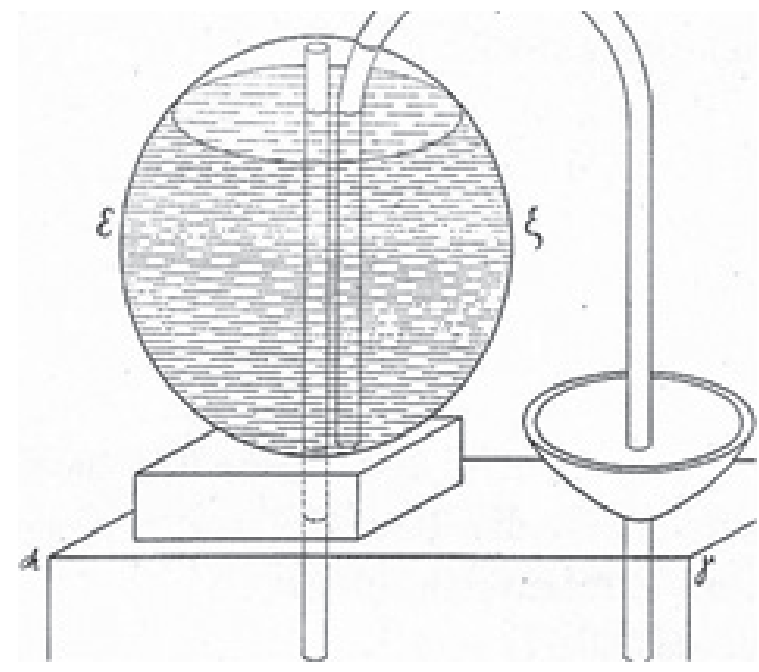

Figura 2. La “gotera” de Filón de Bizancio, prototipo de los primeros termoscopios, ancestros del termómetro.

aparato. Así se observa una relación interesante entre los logros en la física de dos periodos separados por alrededor de 1800 años.

Como mencioné anteriormente, los griegos fueron también los primeros en intentar explicar científicamente los fenómenos atmosféricos y en formular teorías meteorológicas. De hecho, desde la más antigua escuela de los filósofos jónicos, hay pocos filósofos griegos que no se hayan ocupado de alguna rama de la meteorología. Sin embargo, hay que tener presente que esta disciplina abarcaba en aquel entonces un área mucho más amplia que ahora, pues comprendía, además de la meteorología en el sentido moderno, una gran parte de la geografía física y de la astronomía. Los objetos de investigación preferidos de la meteorología eran las precipitaciones, incluidas las periódicas inundaciones del río Nilo, así como la teoría del arcoiris y el origen de los vientos. Pero los meteorólogos también se entregaban a menudo a especulaciones cosmológicas generales que frecuentemente carecían de un fundamento adecuado, resultando ser fantasiosas, fuera de parecer inútiles, si se las juzgaba desde un punto de vista puramente práctico. Este afán por teorizar llegó tan lejos que en los tiempos de Sócrates la meteorología estaba completamente desacreditada. En Atenas se acuñó una nueva palabra - $\mu \varepsilon \tau \varepsilon \omega \rho о \lambda \varepsilon ́ \sigma \chi \eta \varsigma$ en lugar de $\mu \varepsilon \tau \varepsilon \rho o ́ \lambda o \gamma o \varsigma$-, para denominar a alguien que parlotea sobre temas elevados. La comedia de Aristófanes "Las nubes" parece haber sido escrita expresamente como burla a estos teóricos. ${ }^{14}$

\footnotetext{
${ }^{14}$ Aristófanes, Las nubes: Estrepsíades preguntando a Sócrates: Pero dime: ¿quién es el que lanza esos truenos que hacen que me dé el tembleque? Sócrates: Son ellas [las nubes] las que truenan al dar vueltas y más vueltas. Estrepsíades: ¡No te arredras ante nada! ¿Y cómo es eso? Sócrates: Cuando están cargadas de agua en cantidad, rebosantes de lluvia, entonces chocan entre sí pesadamente, se desgarran y explotan con estruendo. Estrepsíades: ¿Y quién es que las fuerza a moverse? ¿No es Zeus? Sócrates: Ni mucho menos: un torbellino etéreo.
}

El azote a los 'forjadores' de hipótesis tuvo un efecto beneficioso, pues se empezó a prestar más atención a los hechos, de modo que un siglo después la investigación meteorológica alcanzó tal nivel que Aristóteles pudo crear por primera vez un cuerpo sistemático de la meteorología cuya autoridad se mantuvo por casi dos milenios.

Desde luego que, si se juzga desde una perspectiva moderna, la meteorología aristotélica se ha tornado hace tiempo obsoleta e inútil. Pero cuando nos trasladamos al espíritu de esa época y se considera el estado general de las ciencias en aquel entonces, se tendrá que admitir que, entre los numerosos escritos de Aristóteles, justo su meteorología representa uno de los trabajos más sobresalientes dignos del más grande pensador sistemático de todos los tiempos ${ }^{15}$.

Me alejaría demasiado del tema, si me dedicara aquí a analizar las ventajas y flaquezas de la meteorología aristotélica. Baste notar que sus eminentes sucesores, como Teofrasto y Posidonio, apenas aportaron al mejoramiento de su sistema teórico, y éste dio más bien pie a innumerables comentarios y paráfrasis. Todos los libros sobre la meteorología que aparecieron hasta el final del siglo XVII e incluso algunos posteriores se basan casi exclusivamente en la concepción aristotélica.

La meteorología tuvo un progreso limitado por parte de los romanos, tal como sucedió con todas las ramas del saber que carecen de valor práctico inmediato. Plinio, Séneca y Lucrecio $^{16}$ aportan pocas cosas nuevas al saber meteorológico

\footnotetext{
${ }^{15}$ Afortunadamente contamos con dos modernas traducciones al español. 1) Aristóteles, 1996: Los meteorológicos. Introducción, traducción y notas de José Luis Calvo Martínez. Alianza Editorial, Madrid. 2) Aristóteles, 1996: Acerca del cielo. Meteorológicos. Introducción, traducción y notas de Miguel Candel. Biblioteca Clásica Gredos, 229. Editorial Gredos, Madrid.

${ }^{16}$ Cayo Plinio Segundo, llamado Plinio el Viejo (23/24-79 d. C.), escritor de obras históricas y la famosa enciclopedia en 37 libros, que reuniera «todo lo que, según los griegos, pertenece a la cultura enciclopédica», incluyendo meteorología y predicción del tiempo, en "oscura mezcla de datos matemáticos y de teorías astrológicas en lo que se refiere a...la meteorología" (véase la edición de Gredos citada a continuación), pero es reacio a aceptar la influencia de los astros sobre la conducta humana. Murió durante la erupción del Vesubio, víctima de éste y su curiosidad científica. Poco sabemos a ciencia cierta de la vida del poeta y filósofo romano Tito Lucrecio Caro (ca. 99-55 a. C), defensor de la doctrina atómica de Epicuro y autor del mejor ejemplo de poesía científica y didáctica de la Antigüedad, el De rerum natura En cuanto a Séneca (4 a. C.- 65 d. C), en materia de "geofísica" nos legó los ocho libros sobre Cuestiones naturales, en los que consagra todos sus esfuerzos a descubrir las causas de diversos fenómenos naturales que habían sido tratados por Aristóteles en su meteorología: crecida del Nilo, vientos, nieve, granizo, terremotos, rayos y truenos etc. Séneca admite la acción de los planetas sobre nuestro destino. En castellano, disponemos afortunadamente de excelentes traducciones modernas de las obras citadas, a saber: Plinio el Viejo, 1995: Historia natural. Libros I-II. Introducción general de Guy Serbat. Traducción y notas de Antonio Fontán, Ana M Moure Casas y otros. Biblioteca Clásica Gredos, 206. Editorial Gredos, Madrid. Séneca, Lucio Anneo, 1979-1980: Cuestiones naturales, 2 vols. Texto revisado y traducido por Carmen Codoñer Merino. Consejo Superior de Investigaciones Científicas, Salamanca. Ver también la reciente edición de Gredos, Séneca, L. A., 2013: Cuestiones naturales. Traducción y notas de José-Román Bravo Díaz. Biblioteca Clásica Gredos, 410. Editorial Gredos, Madrid. Lucrecio, T., 2003: La naturaleza. Introducción, traducción y notas de Francisco Socas, Biblioteca Clásica Gredos, 316. Editorial Gredos, Madrid.
} 
de los griegos, y presumiblemente los escritos perdidos de Nigidio Fígulo y Suetonio Tranquilo ${ }^{17}$ tampoco contenían nada novedoso.

Gracias a Virgilio conocemos algunos nuevos proverbios del tiempo que se originaron en Italia. ${ }^{18}$ Asimismo, Columela ${ }^{19}$, escritor sobre agronomía que poseía una gran hacienda en Cádiz, España, dejó como legado el interesante "Calendarium rusticum”, una especie de almanaque agrícola con indicaciones meteorológicas y fenológicas.

Por el contrario, la extensa colonización de los romanos fue muy apropiada para perfeccionar las hasta entonces vagas ideas sobre las diferencias climáticas de los países. Del mismo modo que la gran expedición militar de Alejandro Magno hacia el interior de Asia e India en su época les proporcionó a los griegos, por primera vez, el conocimiento acerca de los vientos monzónicos, los romanos fueron los primeros en percatarse de la diferencia entre el clima continental y el marítimo. En el siglo II, Minucio Félix, un escritor cristiano-africano, hace la siguiente observación sobre el clima de Gran Bretaña: "Britannia sole deficitur, sed circumfluentis maris tepore recreatur" (Gran Bretaña recibe poca luz solar, pero goza de un clima templado como consecuencia del agua caliente del mar, que rodea al país).

El gran retroceso que se dio después de la caída del Imperio Romano en Europa también trajo consigo un estancamiento prolongado de las ciencias, que apenas se cultivaron dentro de la iglesia. Sin embargo, tampoco en esta época se interrumpió por completo la reflexión sobre los problemas meteorológicos, pues los padres de la iglesia, que escribían múltiples comentarios sobre los siete días de obra de la historia de la creación mosaica, el llamado Hexamerón, añadían frecuentemente a la discusión del primer día de creación largos capítulos sobre las características de la atmósfera y sobre sus cambiantes fenómenos.

A comienzos de la Edad Media, son los grandes enciclopedistas como Isidoro de Sevilla ${ }^{20}$ en España, Beda el Venerable en Inglaterra y Rábano Mauro en Alemania ${ }^{21}$, y otros más, quienes prestaron mayor atención a la meteorología como área del saber; pero también el interés popular en cuestiones meteorológicas debió haberse reavivado para

\footnotetext{
${ }^{17} \mathrm{Cf}$. Sider, en la obra citada en la nota 7. Nigidius Figulus, siglo 1 a. C. Sus escritos en materia de "pronósticos" pueden bien no haber sido parte de una obra dedicada a señales o signos del tiempo. De acuerdo con Aulus Gellius, redactó De augurio privato, que pudo haber ofrecido instrucciones de cómo leer lo cielos no solo en relación con el tiempo sino para toda clase de presagios (omens). Se atribuye a Fígulo un Diarium tonitruale, una elaborada "brontoscopia", o arreglo calendárico, desde junio hasta mayo de qué esperar cada día.

${ }^{18}$ Hellmann se refiere al poema virgiliano Las Geórgicas (29 a. C.), un ditirambo de la vida rural que informa sobre labores agrícolas, y contiene la descripción de signos de tiempo útiles a los agricultores y a los marineros (en el libro I de los cuatro que las componen). Es uno de los poemas más leídos en la época y, más adelante, en el Renacimiento.

${ }^{19}$ Columela, Lucius Junius Moderatus, siglo 1 d. C., “agrónomo” conocido por su De re rustica (Los trabajos del campo), escrito hacia el año 42, amigo de Séneca.
}

ese entonces, pues en el siglo $\mathrm{X}$ apareció en Inglaterra un libro de literatura popular escrito en el idioma anglosajón que instruía sobre asuntos astronómicos y meteorológicos. A juzgar por los ejemplares aún disponibles, este libro debió haber tenido una amplia divulgación, proviniendo probablemente de círculos monacales irlando-escoceses que representaban en aquel tiempo el más alto nivel de la formación europea. De asuntos meteorológicos, el libro trata de forma ingenuamente popular de los vientos, del granizo, de la nieve y del trueno.

Los estudios científicos recibieron un impulso al final del siglo XII, cuando los escritos de Aristóteles, entre ellos también su trabajo sobre la meteorología, llegaron al conocimiento del mundo occidental. Ni siquiera se trataba de los escritos originales en griego, que en Europa occidental en ese entonces no podían ser leídos aún, sino de traducciones del árabe al latín, realizadas en España. El sistema meteorológico formalmente tan bien logrado del estagirita ejerció desde el principio una enorme influencia sobre los escritos de los eruditos y sobre su actividad docente en las recientemente fundadas universidades. Allí se dictaban regularmente clases bajo el título de Meteora que, sin embargo, consistían fundamentalmente solo en la exégesis de la meteorología de Aristóteles. Como mucho se tomaba en consideración la Naturalis Historia de Plinio, o en cátedras especiales se trataba el sistema astrometeorológico de la Antigüedad, que se había ampliado bajo la influencia árabe. En esa época, Alberto Magno escribió en Colonia sus grandes obras meteorológicas (De Meteoris Libri IV y De Passionibus aeris), en las cuales discute y compara el punto el punto de vista de Aristóteles con el de los demás autores, además de agregar sus propias observaciones y percepciones que con frecuencia daban testimonio de una buena capacidad de observación. Igualmente, Vincent de Beauvais en Francia, Thomas de Cantimpré en Bélgica ${ }^{22}$, Ristoro d'Arezzo en Italia y Bartholomaeus de Glanville en

\footnotetext{
${ }^{20}$ Isidoro de Sevilla, nacido en Cartagena, España, vivió entre los años 570 y 636. Por lo tanto, vivió un siglo antes que Beda, en la España relativamente estable de los visigodos. Isidoro fue obispo de Sevilla; escribió obras teológicas inspiradas en Agustín, pero su obra más importante y la más famosa durante la edad media - fueron los Etymologiarum libri. Se trata de una obra que consta de veinte libros....la idea básica de Isidoro es que a través de las etimologías es posible llegar a los significados de las cosas. Como consecuencia de este proyecto, fueron rescatadas del olvido una gran ncantidad de ideas y de concepciones procedentes de autores clásicos, con lo que pudieron transmitirse a las escuelas medievales y ser estudiadas allí. Isidoro es otro de los eslabones que vinculan el mundo clásico con la cultura medieval.

${ }^{21}$ «Fundador de la escuela de Fulda, Hrabano Mauro, autor de un tratado, de clericorum institutione, que propugna el estudio de las artes liberales y de los antiguos filósofos y que le valió el título de praeceptor Germaniae, y de una enciclopedia metódica, el De Universo, cuyos primeros capítulos tratan de Dios, y los últimos, de piedras, entre las cuales está el eco, y de metales.» (Borges, op.cit. nota 2.)

${ }^{22}$ Para la meteorología de Alberto Magno, Vincent de Beauvais y Thomas de Cantimpré (siglo XIII), véase Joëlle Ducos, 1998, La météorologie en français au Moyen Age (XIII ${ }^{e}$ XIV siècles), Honoré Champion, Paris.
} 
Inglaterra dan a conocer ampliamente las ideas aristotélicas en sus grandes obras sobre ciencias naturales que en su mayoría llevan el título de De natura rerum.

Por algún tiempo, el renovado predicamento de Aristóteles reanimó los estudios europeos, con lo cual se adoptaron de manera rígida las enseñanzas del maestro y se negaba todo aquello que no podía encontrarse en sus escritos, lo cual fue tan perjudicial para el verdadero avance en el conocimiento de las cosas, que poco tiempo después hubo un estancamiento absoluto. No obstante, las nuevas ideas que ponían la experiencia y la observación encima de la autoridad Aristotélica, y que, por consiguiente, entraban en grave conflicto con la escolástica, se impusieron paulatinamente. Al respecto me parece bastante digno de consideración que los inicios de las ciencias experimentales modernas coincidieran con la época en que el escolasticismo alcanzó su mayor auge, a saber, en el siglo XIII.

Aún no se ha establecido con total seguridad dónde tuvo origen la nueva ciencia experimental. Muy probablemente fue en Francia e Inglaterra al mismo tiempo, donde los amigos Pierre de Maricourt (Pedro el Peregrino) y Roger Bacon $^{23}$ bien pueden ser considerados como los primeros representantes de este nuevo enfoque. El primero, un noble francés e ingeniero militar de la Picardía, escribió el famoso tratado sobre los imanes y, así como su amigo inglés, realizó varios experimentos ópticos. Aun cuando ninguno de los dos trabajó realmente en el campo meteorológico -exceptuando el arcoiris- su influencia en la ciencia moderna tuvo que haber sido grande. La enérgica lucha de Roger Bacon contra la mera argumentación sin el experimento -argumentum non sufficit, sed experientia-llevó, desde luego, a que también los fenómenos atmosféricos se observaran con mayor precisión y más frecuentemente, en lugar de únicamente interpretar los escritos de los antiguos sabios. El terreno para este fin ya estaba abonado en cierta medida. Pues como ya lo mencioné con anterioridad, en la Antigüedad se realizaron registros meteorológicos, que, a pesar de las frecuentes y extensas interrupciones, es probable que nunca hayan sido abandonados completamente. Pues la costumbre de los analistas romanos de registrar en los anuarios los fenómenos atmosféricos más importantes, principalmente aquellos que exigían sacrificios expiatorios, se transmitió a los cronistas de la Edad Media, cuyas crónicas, siglo tras siglo, tenían cada vez más registros climáticos, hasta que a finales del siglo XIII algunas crónicas tenían tantos registros meteorológicos que es posible reconstruir el carácter general del tiempo de aquel periodo. Es entonces que llega el momento realizar observaciones más sistemáticas. Al inglés William Merle recae la fama de haber llevado el primer diario regular del tiempo en Occidente. Comprende los años entre el 1337 y 1344 y se conserva hoy en día en la Biblioteca Bodleiana de Oxford. A raíz de una sugerencia mía, fue publicada

${ }^{23}$ Pierre de Maricourt (Pedro el Peregrino, conocido también como Pierre de Maricourt), fl. 1269 , y Roger Bacon (ca. 1219-ca. 1292), precursores del método experimental, característico de la Revolución Científica. en impresión facsimilar como uno de los más antiguos monumentos de nuestra ciencia por mi ya fallecido amigo G.J. Symons ${ }^{24}$ en 1891. La muestra en la figura 3 reproduce el final del año 1341.

Aunque en aquel entonces pareciera que el diario meteorológico de Merle fuera el único conocido de su clase, he podido desde entonces descubrir y demostrar que se hicieron observaciones similares en una secuencia casi ininterrumpida del siglo XIV hasta el siglo XVII, es decir que hasta el descubrimiento de los instrumentos meteorológicos más importantes, el tiempo ya había sido observado regularmente en muchos lugares de Europa. Por lo tanto, a lo largo de los milenios se había dado en este ámbito un desarrollo paulatino, a menudo interrumpido, pero en todo caso recurrente, y es así que, de unos registros originales muy esporádicos, especialmente de fenómenos meteorológicos notables, surgen las observaciones periódicas del tiempo atmosférico.

Un poderoso nuevo impulso para la observación y el estudio de los problemas atmosféricos provino al final del siglo XV de un área totalmente diferente. Los grandes descubrimientos de tierra y agua ampliaron de repente el horizonte meteorológico de una forma insospechada. Los navegantes informaron sobre fenómenos atmosféricos que nunca se habían observado y conocieron condiciones climáticas radicalmente diferentes a aquellas de Europa occidental. Los escritos de aquella época nos revelan aún hoy en día la profunda impresión que causaron estas percepciones en los observadores. Luis de Camões, el famoso poeta portugués, describe por primera vez en su poema épico Os Lusiadas trombas de agua que observó a la altura de Guinea y un ciclón al sur del Índico en Madagascar. Así mismo, por el diario de navegación que escribió Cristóbal Colón en el primer viaje a la India occidental, conocemos muy bien

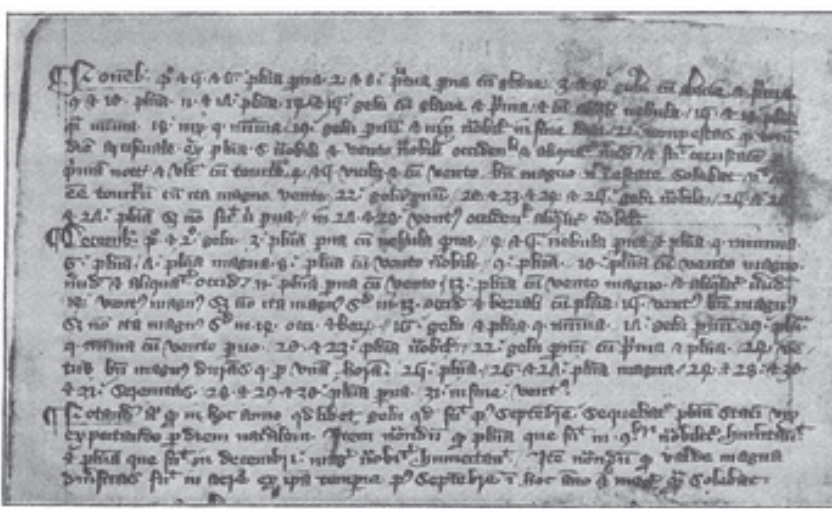

Figura 3. Extracto del diario meteorológico del reverendo William Merle.

${ }^{24}$ George James Symons (1838-1900), organizador de la red pluviométrica de la Gran Bretaña., fundador de la revista Monthly Meteorological Magazine, y gran coleccionista, como Hellmann, de libros de meteorología. Junto con éste uno de los primeros hidrometeorólogos. 
cómo él y su tripulación se vieron consternados por la gran diferencia de las condiciones climáticas y meteorológicas en el Océano Atlántico a uno y otro lado de las Azores.

La ciencia de los vientos fue la que más avanzó gracias a estas nuevas observaciones. En la rica literatura náutica del siglo XVI, que se dio principalmente en España y Portugal, podemos comprobar de la mejor manera estos avances, hasta que en el año 1622 Francis Bacon escribiera por primera vez desde la Antigüedad, un libro especial sobre los vientos ${ }^{25} \mathrm{e}$ hiciera el intento de indagar sobre la circulación general de la atmósfera.

Entretanto, la ciencia experimental, cuyos inicios en el siglo XIII esbocé hace un momento, se había desarrollado al punto de que a mediados del siglo XVII se inventaron

${ }^{25}$ Véase De ventibus (1622) en la reciente edición crítica de las obras completas de Bacon, 2007: The Oxford Francis Bacon, Vol. XII. The Instauratio magna, Part III: Historia naturalis et expermientalis: Historia ventorum and Historia vitae \& mortis. Edited with Introduction, Notes, Commentaries, and Facing-Page Translations by Graham Rees with Maria Wakely. The Clarendon Press, Oxford. los instrumentos meteorológicos más importantes. ${ }^{26}$ Italia tuvo la gloria de ser el lugar donde nació la meteorología instrumental, cuya cuna es Florencia.

No quiero dejar pasar esta oportunidad para homenajear a Evangelista Torricelli, el inventor del barómetro, cuyos 300 años se celebran en Italia en este día (25 de octubre). La invención de los instrumentos meteorológicos fue el primer paso para que la meteorología se convirtiera paulatinamente de un campo del saber en una ciencia. Con esto llegan a su fin los inicios de la meteorología.

\section{Contribución de los autores}

ALPW la traducción, SBC, la supervisión y corrección de la traducción, y JP la introducción, notas y revisión técnica de la traducción.

${ }^{26}$ Véase Hellmann,1920, Beiträge zur Erfindungsgeschichte der meteorologischen Instrumente (Contribuciones a la historia de la invención de instrumentos meteorológicos). Abhandlungen d. Preuß. Akad. d. Wiss. Berlin, Jg. 1920. Phys.-Math. Klasse Nr. 1., 60 S. 